\title{
Morphological and crystallographic analysis of kidney stones in Eastern Morocco
}

\author{
Bouchra El Guerrouj ${ }^{1}$, Mohamed Bouhrim ${ }^{2}$, Noureddine Bencheikh ${ }^{2}$, Hamada Imtara ${ }^{3}$, Loubna \\ Kharchoufa $^{2}$, Hayat Ouassou ${ }^{2}$, Yassamine Bentata ${ }^{4}$, Michel Daudon ${ }^{5}$ and Mohammed Melhaoui ${ }^{1}$ \\ ${ }^{1}$ Laboratory of Water Environment and Sustainable Development, Faculty of Sciences, \\ Mohammed First University, Oujda, Morocco \\ ${ }^{2}$ Laboratory of Physiology, Genetics, and Ethnopharmacology, Department of Biology, Faculty of Sciences, \\ Mohammed First University, Oujda, Morocco \\ ${ }^{3}$ Faculty of arts and sciences, Arab American University Palestine, Po. box 240, Jenin, Palestine \\ ${ }^{4}$ Laboratory of Epidemiology, Clinical Research, and Public Health, Faculty of Medicine and \\ Pharmacy of Oujda \\ ${ }^{5}$ Department of physiology-functional explorations, Tenon Hospital, Paris, France
}

\begin{abstract}
Renal lithiasis is a common disease in the eastern region of Morocco, as it threatens the public health because of its repetitive nature as well as the complications it can cause in the kidney.
\end{abstract}

Objective: This study aims to investigate the morphological, molecular, and crystallographic characteristics of kidney stones affecting patients, specifically in the eastern region of Morocco.

Methods: Morphological and constitutional analyses of 239 renal lithiases made it possible to identify the various crystalline forms present, to assess the structure of the stones and to determine the composition of their nucleation zone.

Results: It appears that calcium oxalate is the main component of the analyzed stones. It represents $70.5 \%$ of the stones, which $55.5 \%$ are majority whewellite and $15 \%$ in weddellite. In comparison, calcium phosphates and magnesium (carbapatite and struvite) are the majority in only $8.9 \%$ of cases, uric acid in $19.8 \%$ of stones, and ammonium acid urate in $0.8 \%$. Calcium oxalate is predominant in the core of $52.5 \%$ of the stones, carbapatite in $24.1 \%$, and uric acid in $20.3 \%$ and struvite in $2.9 \%$. Most of the stones analyzed consist of a mixture of different crystalline constituents. Only $9.3 \%$ of the stones analyzed have a homogeneous composition. Several types of associations were identified, the main ones being whewellite-carbapatite and whewellite-weddellite-carbapatite. Conclusion: The present study shows that calcium oxalate is the most common compound in the samples studied, followed by the uric acid compound.

Keywords: renal lithiasis; stones; morphology; crystallography; infrared analysis; Morocco.

\section{Introduction}

Kidney stone (Urolithiases) is the process in which stones have emerged inside the kidney that could be a result of many factors including climate, geographical location, occupation, diet, social class, genetic factors and crystallization of certain chemicals that are supersaturated in the urine ${ }^{1,2}$. Lithiasis is a real problem where is estimated to affect $12 \%$ of the world population with its prevalence in women more than men ${ }^{1,3}$

In some developed countries such as the United States and France, they carried out important research on lithiasis as early as 1976 4. Meanwhile, in developing

*Corresponding author: Hamada Imtara

Email address: hamada.tarayrah@gmail.com

DOI: http://dx.doi.org/10.13171/mjc10502005261409hi countries where the health sector is quite limited, only a few cases such as Algeria and Tunisia, where research has been carried out on the analysis of kidney stones in adults and children since 1995,6. In Morocco, a study on kidney stones in about 453 children have shown that calcium oxalate was the main component in $51.6 \%$ of the stones, followed by struvite $(18.1 \%)$, ammonium urate $(9.5 \%)$ and carbapatite $(9 \%)^{7}$.

Urinary/renal calculi is a complex concretion and the key element in the etiological diagnosis of lithiasis disease, and the process of identifying the chemical components of the latter helps in understanding the

Received March 19, 2020

Accepted April 20, 2020

Published May 26, 2020 
factors and the way that leads to the formation of these pathology ${ }^{8,9}$. Calculi are very diverse; some

multi-recurrent forms are responsible for significant morbidity and sometimes impaired renal function, which can lead to end-stage renal disease ${ }^{10,11}$.

The investigations of the types of the lithiasis must include morpho-constitutional analysis and biological exploration guided by clinical and radiological data as well as by the physical analysis's results. However, this rational approach to lithiasis is the only way possible that led to defining the therapeutic measures adapted to the prevention of recurrences 12,13 Therefore, this study was conducted to determine the renal calculi (morphology, chemical composition) in eastern Morocco as well as to classify the calculi according to the morpho-constitutional classification and determine the most frequent constituents.

\section{Material and Methods}

\subsection{Material and reagents}

The materials and reagents used in this study are:

- A magnifying lens for the study of organoleptic characteristics.

- A Fourier infrared spectrophotometer (IRTF) covering the spectral range from 2.5 to $50 \mathrm{um}$.

- A pellet mould for the preparation of $13 \mathrm{~mm}$ diameter pellets.

- A pellet mold and accessories for the production of pastille of $0.5-1 \mathrm{~mm}$ diameter.

- A perforated metal disc (diameter of $13 \mathrm{~mm}$, and diameter of the perforation of $2 \mathrm{~mm}$ ).

- A beam condenser for micropellets.

- A vacuum pump.

- Pure and dry potassium bromide.

\subsection{Ethical}

The patient personal data are strictly confidential. Each patient had a code assigned to them, and a record was established to identify the different patients. This register was only accessible to the principal investigator. Data collection and data entry was done anonymously using this code.

\subsection{Morphological and structural analysis}

The study was based on the morphological and constitutional analysis of 239 renal calculi collected during the beginning of March 2011 until the end of February 2016. These renal calculi are common among adult patients with Lithiasis located in different regions of Eastern Morocco. In this study, patients who had renal lithiasis was diagnosed and confirmed by the departments of urology or nephrology in private or public health sector throughout the region. A lithiasis questionnaire supported each calculus that it is believed to cover every aspect of the latter.
Furthermore, In the present study, only renal lithiasis was taken into consideration, ureter and bladder lithiasis, as well as children urolithiasis, were kept for further studies. The Protocol analysis method used in the experimental analysis of this work, is based on Daudon study ${ }^{14}$, it involves the following steps:

\section{1- Calculi cleansing}

2- A morphological examination of the calculi with the unaided eye and the binocular magnifying loupes to determine the morphological type of the surface, section, and nucleus.

3- A selective and complete infrared spectrophotometry study, which includes the following analysis; First, a qualitative and sequential quantitative analysis of the nucleus at the surface; second, an overall powder analysis of the calculi which aims to determine the relative proportion of various constituents present in the calculi.

4- Determination of the color of the individual zones.

\subsubsection{Study of external morphological characteristics}

The objective of the morphological and structural analysis was to specify, using binocular magnifying loupes, the appearance of the nucleus, and the depth of the calculi`s layers. In the form of a morphological type according to the classification developed by Daudon. With a sampling of all representative surface and deep areas of the structure. Calculi were cleaned with distilled water and dried at room temperature for 24 hours before analysis. The purpose of this process is to recognize the general organoleptic characteristics of the calculi as well as the shape and dimension with the naked eye, and the outward symptoms with binocular magnifying loupes. In addition to that, this process also includes the following essential steps:

1- Examination of aspect: homogeneous, heterogeneous, nipple, spicule, bumpy, budding, smooth, rough, porous, cracked, chipped.

2- Examination of the crystalline, microcrystalline, or amorphous character.

3- The search for structural particularity, deposits, and weather an umbilication is present or not, incomplete layers, cavities, side faces to other calculations.

\subsubsection{Calculi section study}

At section level we have looked for the nucleation zone by appreciating all the details of the internal structure, a cut of the calculi was carried out at the level of the most swollen parts, we appreciated the hardness, fragile character, the compact or loose structure, concentric or radial, unorganized, the concentricity in layers alternates or not, we also appreciated the crystalline, microcrystalline or amorphous, homogeneous or heterogeneous nature, and whether a diffusion in the nucleation zone exists, of a nucleus well individualized, or a point of convergence of crystallization, or a foreign body at 
the center of the structure (surgical ligation, a fragment of the probe, hair...) or even convergence towards an empty center (initial protein nucleus secondarily retracted) We looked for the presence or not of deposits or internal, localized or scattered layers as well as the presence of incomplete layers. Several perpendicular sections of the calculi were occasionally requisite to find the nucleation zone. The color of the different structurally individualized zones was noted. Finally, the separate morphological typing of the surface, nucleus, and section was determined by the classification of Daudon, and this is through the classification table, which includes the description of the superficial and internal morphological characters for each type.

\subsection{Study of the molecular and crystalline composition of renal calculi}

\subsubsection{Sample collection for infrared analysis}

Samples for infrared analysis were chosen according to the morpho structural characteristics of the calculi. This step consisted of analyzing the calculi of the nucleus sequentially at the surface using infrared spectrophotometers.

The sampling was carried out in the form of pellets obtained by sampling small quantities of product from each calculi area.

\subsubsection{Qualitative determination}

Selective sampling was carried out of all the structural zones characteristic of the surface and the section observed with both the naked eye and the binocular magnifying glass, the number of samples was conditioned by the degree of heterogeneity of the calculi structure.

\subsubsection{Quantitative determination}

A fragment representative of the whole structure of the calculi where sometimes whole calculi have been sprayed to obtain a global powder on which the proportions of the different constituents are determined so that the quantification will be complete and will cover all the calculi components.

\subsubsection{Preparation technique}

A small fraction of each test area (1-2 mg) was pulverized and then crushed and diluted in the ratio of $0.5-2 \%$ within $100 \mathrm{mg}$ of very dry potassium bromide, with an agate mortar to obtain a homogenized mixture. The powder was placed in a pellet mold and pressed (10 tonnes for 2 or $3 \mathrm{~min}$ ) into pellets with a diameter of $13 \mathrm{~mm}$ under a vacuum pump. Once the pellet is prepared, it is placed in the sample beam of the spectrophotometer. The spectrum is recorded. The spectra obtained enabled the mineralogical identification of the body or bodies by reference to a spectrum bank. Once the component identified, it was confronted with morphological analysis. Finally, each calculi was sprayed in its entirety, and then the homogenized powder was analyzed by an infrared spectrophotometer to determine the overall composition of calculi. For tiny areas, especially small cores, the micro-blasting technique was applied. The powder obtained after homogeneous mixing of the sample with potassium bromide is introduced into the central perforation of the disc previously arranged in the matrix. The pasting itself was carried out according to the same protocol as that described for the conventional technique. At the end of the compression, the disc, which must have the transparent pellet in its center is fixed on a capacitor capable of concentrating up to 8 times the beam analyzed by the detector. To compensate for the loss of energy caused by the absorption of the metal disc.

\subsection{Statistical analysis}

The analysis of the kidney calculi was followed by a statistical analysis using the khi 2 test for the comparison of the numbers, the student test, and the study of the variances for the comparison of the means. The calculi were classified according to their main component.

\section{Results and Discussion}

\subsection{Frequency of the different types of calculi depending on age and sex}

The age of the lithiasis patients who participated in the study is between 18 and 77 years with an average of 37.5 years; among the 239 calculi analyzed, $69 \%$ are males origin, and $31 \%$ are of female origin (overall ratio $\mathrm{M} / \mathrm{F}=1.8$ ). The series of calculi collected from the lithiasis of the Eastern region of Morocco shows a male predominance, these results are consistent with the data found in Thailand ${ }^{15}$, Italy ${ }^{16}$, the United States ${ }^{17}$, Japan ${ }^{18}$, and also in Tunisia ${ }^{19}$. Besides, $88 \%$ of these calculi were expelled spontaneously. In comparison, only $12 \%$ were surgically extracted, and $66 \%$ of the renal calculi in the series are from the left kidney, and $32 \%$ are from the right kidney.

\subsection{Morphological and crystallographic analysis of renal calculi \\ 3.2.1. The main mineralogical constituents in the calculi of the series studied}

The morpho-constitutional analysis of the various calculi of the series showed that the calcium oxalate is the main dominant component of the calculi; it forms the majority in $70.5 \%$ of the calculi, as for the uric acid it is in $19.8 \%$ of cases, while calcium phosphates and magnesium are present in $8.9 \%$ of cases.

The morpho-constitutional analysis of the various calculi of the series showed that the calcium oxalate is the main dominant component of the calculi, so that it plays a vital role in the genesis and growth of the majority of the lithiasis. The overall frequency of mineralogical distribution of calcium oxalate, calcium and magnesium phosphates, and uric acid are of the same order of magnitude as those observed in France ${ }^{20}$, in eastern India ${ }^{21}$, and in the United 
States ${ }^{22}$, and other Maghreb countries (Table 1). However, there are some differences with France, notably a reversal in the proportions of phosphatic and uric acid calculi, the latter being more frequent in our series. As for the recently published Algerian series, it is characterized by a relatively high proportion of phosphatic calculi $(26,6 \%)$, suggesting a significant contribution of infectious processes to the development of lithiasis, a phenomenon that we do not find in our sample.

Table 1. Distribution of the three main mineralogical components of the calculi in Morocco, Algeria ${ }^{23}$, and France ${ }^{20}$.

\begin{tabular}{|c|c|c|c|}
\hline Majority constituent (\%) & Morocco & Algeria & France \\
\hline Calcium oxalate & 70,5 & 61,2 & 66 \\
\hline Uric acid & 19,8 & 8,1 & 10,1 \\
\hline Phosphates & 8,9 & 26,6 & 19,4 \\
\hline
\end{tabular}

3.2.2. Frequency of the constituents in the overall chemical composition of the calculi and frequency of the majority constituents in the calculi and the nucleus

The frequency of the constituents in the chemical composition of calculi and nucleus is shown in Tables 2 and 3. The most frequently observed constituent is whewellite, which is present in $89.4 \%$, represents the main component in $55.5 \%$ of the cases, and constitutes the nucleus in $39.8 \%$ of the calculi analyzed. Moreover, weddellite is present in $38.5 \%$ of the cases, the majority in $15 \%$ of the calculi, and $12.7 \%$ of the nuclei. These results agree with the results found in France ${ }^{2,24}$, Italy ${ }^{16}$, Israeli ${ }^{25}$, and Tunisia ${ }^{26}$. The monohydrate or whewellite form is oxalate dependent and attached to hyperoxaluria. The monohydrate or whewellite form is a dependent oxalate, is attached to hyperoxaluria.

In contrast, the dihydrate or weddellite form is dependent on calcium and is attached to hypercalciuria. The monohydrate or whewellite form is dependent on oxalate. These results point towards a moderate hyperoxaluria flow, of nutritional origin, or towards hyperoxaluria of concentration caused by insufficient diuresis ${ }^{25,27}$. Carbapatite is found in $67.8 \%$ of the samples examined; it is the majority in only $3.4 \%$ of the cases and constitutes $24.1 \%$ of the nuclei. The struvite, which is present in $9.7 \%$ of cases, is the majority in $5.5 \%$ of calculi and $2.9 \%$ of nuclei. It should also be noted that the protein content was more or less relevant; the proteins were present in $29.6 \%$ of the calculi analyzed at proportions, not exceeding $10 \%$ of the overall composition of the calculi. The protein matrix plays a secondary role in most oxalic calculi; this matrix is often weak in weddellite type Ia calculi. Still, it is relatively abundant in whewellite structures, or it plays a role of grain joint mainly in type Ia calculi, which carry papillary umbilication ${ }^{13}$.

Table 2. Frequency of constituents in the overall chemical composition of the calculi.

\begin{tabular}{|c|c|}
\hline Constituent & Frequency (\%) \\
\hline Calcium oxalate & \\
- Whewellite & 89.4 \\
- Weddellite & 38.5 \\
\hline Calcium phosphates & \\
- Carbapatite & 67.8 \\
- PACC & 11.4 \\
\hline - Whitlockite & 4.2 \\
\hline Struvite & 3.8 \\
\hline Purines & 9.7 \\
\hline - Unhydrous uric acid & \\
\hline - Ammonium acid urate & 24.1 \\
\hline - Sodium acid urate & 10.6 \\
\hline Trimagnesium Phosphate Pentahydrate & 4.2 \\
\hline Protein & 0.4 \\
\hline
\end{tabular}

A majority calculus of uric acid dihydrate $(0.8 \%)$ suggests metabolically active lithiasis of recent formation by hyperuricemia and/or urinary acid $\mathrm{pH}$.
While a very majority or even pure lithiasis of anhydrous uric acid (19\%) generally supposes an inevitable delay between the formation of stones and 
its clinical expression, the time necessary for the crystalline conversion of the dihydrate form, which is most easily, and a form which can be rapidly crystallized in urine into the thermodynamically more stable anhydrous form. These data can help us to reconstruct the history of lithiasis, to appreciate more precisely the lithogenic factors, and to define the appropriate prophylactic measures ${ }^{28}$.

Table 3. Frequency of majority constituents in the calculi and the nucleus.

\begin{tabular}{|c|c|c|}
\hline Majority constituents & Frequency in the calculi $(\boldsymbol{\%})$ & Frequency in the nucleus $(\boldsymbol{\%})$ \\
\hline Whewellite & 55.5 & 39.8 \\
\hline Weddellite & 15 & 12.7 \\
\hline Anhydrous uric acid & 19 & 20.3 \\
\hline Uric acid dihydrate & 0.8 & 0 \\
\hline Ammonium acid urate & 0.8 & 0 \\
\hline Carbapatite & 3.4 & 24.1 \\
\hline Struvite & 5.5 & 2.9 \\
\hline
\end{tabular}

On the other hand, the morpho-constitutional analysis showed that the nucleus is either diffuse, does not differ from the central mass of the calculation, or it is different from this one and then presents specific characteristics. In the latter case, the main component of the nucleus is calcium oxalate in $52.5 \%$ of cases, carbapatite in $24.1 \%$ of cases, uric acid anhydrous in
$20.3 \%$ of cases, and struvite in $2.9 \%$ of cases. The various cases encountered during the study show that the composition of the nucleus may be identical to that of the central mass of the calculation or, on the contrary, it is very different, which proves that there may be a difference between the crystalline growth factors and nucleation factors (Table 4).

Table 4. Major constituent observed in the nucleus as a function of the main constituent of the calculi.

\begin{tabular}{|c|c|c|}
\hline $\begin{array}{c}\text { Majority constituent } \\
\text { Of the calculi }\end{array}$ & $\begin{array}{c}\text { Majority constituent } \\
\text { of the nucleus }\end{array}$ & Frequency (\%) \\
\hline Calcium oxalate & Whewellite & 39.8 \\
& Weddellite & 12.7 \\
\hline \multirow{2}{*}{ Phosphates } & Uric acids & 2.5 \\
& Carbapatite & 18.2 \\
\hline Uric acid & Carbapatite & 5.9 \\
& Struvite & 2.9 \\
\hline
\end{tabular}

Our findings showed that the crystalline phases detected in the calculi present a similarity and big difference between our series, French series, and Algerian series. Whewellite predominates in $55.5 \%$ of Moroccan calculi, in $48.1 \%$ of Algerian calculi, and $42.8 \%$ of French calculi, while weddellite predominates in $15 \%$ of Moroccan calculi, in $13.1 \%$ of Algerian calculi and $23.2 \%$ of French calculi. Whewellite being oxalic-dependent and weddellite essentially calcium-dependent, such a difference in distribution suggests that the calcium intakes are different between the Moroccan population and the French population. In contrast, there is a rapprochement between the Moroccan population and the lithiasis population of the West Algerian. The struvite was detected, as shown in the following Table 3 in $9.7 \%$ of the Moroccan calculi. However, it was found in $20.6 \%$ of the Algerian calculi. This difference attests that urinary tract infections by ureteric germs are more frequently involved in the development of calculi in Algeria than that in Morocco ${ }^{29}$. Another difference concerns the frequency of ammonium acid urate. It was identified only in $4.2 \%$ of Moroccan calculi, and in $12.2 \%$ of Algerian calculi, this difference seems significant. It is likely that the eating habits in Morocco, mainly the regular consumption of dairy products, have contributed to limit the formation of ammonium acid urate lithiasis, which is usually due to a protein and phosphorus deficiency ${ }^{20}$.

In a study carried out in Federal Germany, it was shown that calcic lithiasis are dominant, and the most common $75 \%$ followed by uric acid calculi $11 \%$ cystine is observed only in $1 \%$ of cases, which is similar to results obtained in other studies ${ }^{30}$. A representative study of Japan, conducted in Osaka, was showed that calcium calculi account for $85.5 \%$ of the calculi, namely calcium oxalate, hydroxyapatites, or the mixture of both. For the other types of calculi were infection calculi such as struvite, carbon apatite, uric acid, and cystine, represent only $14.5 \%{ }^{31}$. Indeed, all the studies published on the analysis of renal lithiasis have shown that calcium oxalate 
monohydrate COM is the most common crystal, and the major component of most calculi, apatite, is the second common crystal. Dihydrate Calcium oxalate COD is the third ${ }^{20}$. During the analysis, we encountered two main forms of calcium oxalate, calcium monohydrate oxalate, which is the majority constituent of the calculi, and calcium oxalate dihydrate, which is present in a significant proportion in the calculi. While calcium oxalate trihydrate is remarkably absent, these results correlate with the results obtained in the major series of calculi analyzed in the European countries and Japan. Calcium oxalate is a form of calcium oxalate that is very rare; it is more unstable than weddellite and usually turns into whewellite within a few hours, which it is close to through the crystalline system ${ }^{18,24}$.

\subsubsection{Distribution of the calculi according to the constituents and frequency of the different associations encountered}

Besides, the infrared analysis made it possible to identify 1 to 6 mineralogical constituents by calculi, as shown in Table 5. We considered as heterogeneous even the calculi, which contained a single chemical species and which was present in several crystalline forms as well as the calculi containing a deficient proportion of calcium phosphates, less than $3 \%$. The combinations of $2,3,4,5,6$ were observed in $33.9 \%$, $43.2 \% 11 \%, 2.1 \%$ and $0.4 \%$ respectively. This correlates with literature evidence that the calculi are generally mixed in $80 \%$ of cases and is composed of two or more crystalline species ${ }^{9}$. Among the different combinations encountered during the study, the most common were mixtures of whewellite-carbapatite $(19.5 \%)$ and whewellite-weddellite-carbapatite. $(27.1 \%)$ with a difference in the number of constituents from one calculi to another (Table 5).

Table 5. Distribution (\%) of the calculi according to the constituents and frequency (\%) of the different associations encountered.

\begin{tabular}{|c|c|c|c|}
\hline $\begin{array}{l}\text { Number of } \\
\text { constituents }\end{array}$ & Frequency $(\%)$ & $\begin{array}{l}\text { Nature of the } \\
\text { constituents }\end{array}$ & Frequency $(\%)$ \\
\hline 1 & 9.3 & $\begin{array}{c}\mathrm{Wh} \\
\mathrm{AU}_{0}\end{array}$ & $\begin{array}{l}6.3 \\
2.9\end{array}$ \\
\hline 2 & 33.9 & $\begin{array}{c}\mathrm{Wh}+\mathrm{PCa} \\
\mathrm{Wh}+\mathrm{AU}_{0} \\
\mathrm{Wh}+\mathrm{Wd} \\
\mathrm{AU}_{0}+\mathrm{AU}_{2} \\
\mathrm{Wd}+\mathrm{PCa}\end{array}$ & $\begin{array}{l}19.5 \\
6.7 \\
3.8 \\
2.9 \\
0.8\end{array}$ \\
\hline 3 & 43.2 & $\begin{array}{c}\mathrm{Wh}+\mathrm{Wd}+\mathrm{PCa} \\
\mathrm{Wh}+\mathrm{AU}_{0}+\mathrm{AU}_{2} \\
\mathrm{Wh}+\mathrm{PCA}+\mathrm{Wit} \\
\mathrm{Wh}+\mathrm{PCa}+\mathrm{PAM} \\
\mathrm{Wh}+\mathrm{AU}_{0}+\mathrm{UrAm}\end{array}$ & $\begin{array}{l}27.1 \\
7.2 \\
1.2 \\
1.7 \\
0.8\end{array}$ \\
\hline 4 & 11 & $\begin{array}{c}\mathrm{Wh}+\mathrm{Wd}+\mathrm{PCa}+\mathrm{PACC} \\
\mathrm{Wh}+\mathrm{PCa}+\mathrm{PAM}+\mathrm{PACC} \\
\mathrm{PCa}+\mathrm{PACC}+\mathrm{Wit}+\mathrm{New} \\
\mathrm{Wh}+\mathrm{PCa}+\mathrm{PACC}+\mathrm{Wit} \\
\mathrm{PCa}+\mathrm{PAM}+\mathrm{PACC}+\mathrm{UrAm} \\
\mathrm{Wh}+\mathrm{Wd}+\mathrm{PCa}+\mathrm{PAM} \\
\mathrm{Wh}+\mathrm{Wd}+\mathrm{PCa}+\mathrm{UrAm} \\
\mathrm{Wh}+\mathrm{PCa}+\mathrm{AU} \mathrm{U}_{0}+\mathrm{PACC} \\
\mathrm{PCa}+\mathrm{PAM}+\mathrm{PACC}+\mathrm{UrNa} \\
\mathrm{PCa}+\mathrm{PAM}+\mathrm{PACC}+\mathrm{New}\end{array}$ & $\begin{array}{l}3.4 \\
1.7 \\
0.4 \\
1.2 \\
0.8 \\
0.8 \\
1.2 \\
0.4 \\
0.4 \\
0.8\end{array}$ \\
\hline 5 & 1.2 & $\begin{array}{c}\mathrm{Wh}+\mathrm{PCa}+\mathrm{PAM}+\mathrm{New}+\mathrm{UrAm} \\
\mathrm{Wh}+\mathrm{PCa}+\mathrm{PAM}+\mathrm{Wit}+\mathrm{New} \\
\mathrm{Wh}+\mathrm{Wd}+\mathrm{PCa}+\mathrm{AU}_{0}+\mathrm{AU}_{2}\end{array}$ & $\begin{array}{l}0.4 \\
0.8 \\
0.8\end{array}$ \\
\hline 6 & 0.4 & $\mathrm{Wh}+\mathrm{PCa}+\mathrm{PAM}+\mathrm{PACC}+\mathrm{Wit}+\mathrm{UrAm}$ & 0.4 \\
\hline
\end{tabular}

Wh: Whewellite, Wd: Weddellite, $\mathrm{AU}_{0}$ : Anhydrous Uric acid, $\mathrm{AU}_{2}$ : Uric acid dihydrate; PCa: carbapatite, PAM: struvite, New: new beryte, Wit: whitlockite, UrAm: Ammonium acid urate, UrNa: Sodium urate, PACC: amorphous calcium phosphate carconate.

The association of two crystalline species suggests a double biochemical anomaly, that is, a multifactorial etiology ${ }^{28}$. The presence of $\mathrm{C} 1$ and $\mathrm{C} 2$ in a calculi is often associated with mixed disorders and pathological disorders in the kidney (hyperoxaluria and hypercalciuria). The intervention of other urinary factors (magnesium, citrates, etc.) inhibitors or promoters of any crystalline phase, type II C2 calculi appear dependent calcium, the abundant presence of less than $25 \%$ calcium phosphate in oxalic- calculi. It 
should be mentioned that an additional pathology is involved in either phosphorus excretion or $\mathrm{pH}^{13}$.

\section{Conclusion}

The morpho-constitutional analysis showed the highest number of lithiasis have a heterogeneous molecular and crystalline composition, with a predominance of calcic lithiasis. In contrast, a limited number of the calculi of the series $(9.3 \%)$ are pure and have a homogeneous overall composition and are mainly calculi of whewellite and uric acid. The infrared analysis enabled us to identify one to six mineralogical constituents by calculi. The most common combinations are two or three constituents, the most commonly encountered being whewellitecarbapatite and whewellite-weddellite-carbapatite. Calcium oxalate lithiasis in both monohydrate and dihydrate forms has the highest frequency of all calculi. They are the main form of renal lithiasis in both males and females in the population. This first study confirms that in Eastern Morocco, as in other countries, calcium oxalate is the main lithiasis constituent. The frequency of uric acid lithiasis follows the frequency of calcium oxalate lithiasis.

Infection lithiasis has a shall low frequency, which demonstrates that infectious processes rarely intervene in the development of renal lithiasis in the eastern region.

\section{References}

1- T. Alelign, B. Petros, Kidney stone disease: an update on current concepts, Advances in urology, 2018, 2018, 1-12.

2- M. López, B. Hoppe, History, epidemiology and regional diversities of urolithiasis, Pediatric Nephrology, 2010, 25, 49-59.

3- A. Tiwari, V. Soni, V. Londhe, A. Bhandarkar, D. Bandawane, S. Nipate, An overview on potent indigenous herbs for urinary tract infirmity: Urolithiasis, Asian Journal of Pharmaceutical and Clinical Research, 2012, 5, 7-12.

4- G. C. Curhan, Epidemiology of Stone Disease, Urol Clin North Am, 2007, 34, 287-293.

5- A. Kamoun, M. Daudon, N. Kabaar, R. Dhaoui, S. Ben Ammar, Facteurs étiologiques de la lithiase urinaire de l'enfant en Tunisie, Progrès en urologie (Paris), 1995, 5, 942-945.

6- D. Harrache, Z. Mesri, A. Addou, A. Semmoud, B. Lacour, Analyse des calculs urinaires de l'adulte dans l'Ouest Algérien par spectroscopie infrarouge à transformée de Fourier, L'Eurobiologiste (Paris), 1997, 31, 11-16.

7- F. Meiouet, S. El Kabbaj, M. Daudon, Pediatric urolithiasis in Morocco: Composition of 432 urinary calculi analyzed by infrared spectroscopy, Progres en Urologie, 2019, 29, 173-182.

8- M. Daudon, A. Valognes, C. Hennequin, P. Jungers, Importances de l'analyse morphoconstitutionnelle des calculs et des cristaux urinaires pour le diagnostic étiologique et le suivi thérapeutique de la maladie lithiasique, Spectra biologie, 1992, 5, 33-51.

9- M. Daudon, R. J. Réveillaud, Typage morphologique des calculs oxalocalciques et données étiopathogéniques, Annales d'urologie, 1985, 19, 299-308.

10-M. T. Keddis, A. D. Rule, Nephrolithiasis and loss of kidney function, Curr Opin Nephrol Hypertens, 2013, 22, 390-396.

11-E. W. Vahlensieck, D. Bach, A. Hesse, A. Strenge, Epidemiology, pathogenesis and diagnosis of calcium oxalate urolithiasis, International urology and nephrology, 1982, 14, 333-347.

12- M. Daudon, R. J. Réveillaud, L'analyse des calculs urinaires en routine importance pour le diagnostic étiologique de la lithiase, Fiches Pra Biol, 1985, 3, 43-49.

13-M. Daudon, R. J. Réveillaud, Whewellite et weddellite: vers des éthiopathogénies différentes: intérêt dy typage morphologique des calculs, Néphrologie (Genève), 1984, 5, 195-201.

14-M. Daudon, Plaidoyer pour une meilleure exploration des lithiases rénales, L'Eurobiologiste, 1997, 31, 17-35.

15-P. Tosukhowong, C. Boonla, S. Ratchanon, M. Tanthanuch, K. Poonpirome, P. Supataravanich, T. Dissayabutra, K. Tungsanga, Crystalline composition and etiologic factors of kidney stone in Thailand: update 2007, Asian Biomedicine, 2007, 1, 87-95.

16-M. L. Giannossi, G. Mongelli, F. Tateo, V. Summa, Mineralogical and morphological investigation of kidney stones of a Mediterranean region (Basilicata, Italy), Journal of X-Ray Science and Technology, 2012, 20, 175-186.

17-H. Patel, Kidney Stones 2019: Epidemiology, Clinical Pathophysiology and Treatment, 2019. https://hdl.handle.net/2152.5/6771.

18- R. Z. Hossain, Y. Ogawa, S. Hokama, M. Morozumi, T. Hatano, Urolithiasis in Okinawa, Japan: A relatively high prevalence of uric acid stones, International Journal of Urology (2003), 2003, 10, 411-415.

19-H. Bouzidi, D. de Brauwere, M. Daudon, Does urinary stone composition and morphology help for prediction of primary hyperparathyroidism? Nephrology Dialysis Transplantation, 2011, 26, 565-572.

20-M. Daudon, Epidemiology of nephrolithiasis in France, Annales d'Urologie, 2005, 39, 209-231.

21-P. Chatterjee, A. Chakraborty, A. K. Mukherjee, Phase composition and morphological characterization of human kidney stones using IR spectroscopy, scanning electron microscopy and X-ray Rietveld analysis, Spectrochimica Acta Part A: Molecular and Biomolecular Spectroscopy, 2018, 200, 33-42.

22-P. M. Patel, A. M. Kandabarow, A. Druck, S. Hart, R. H. Blackwell, A. Kadlec, A. Farooq, T. M. T. Turk, K. G. Baldea, Association of 
Impaired Renal Function With Changes in Urinary Mineral Excretion and Stone Composition, Urology, 2020. doi:10.1016/j.urology.2020.03.023.

23-D. Harrache, Z. Mesri, A. Addou, A. Semmoud, B. Lacour, Analyse des calculs urinaires de l'adulte dans l'Ouest Algérien par spectroscopie infrarouge à transformée de Fourier, L'Eurobiologiste (Paris), 1997, 31, 11-16.

24-R. C. Walton, J. P. Kavanagh, B. R. Heywood, P. N. Rao, Calcium oxalates grown in human urine under different batch conditions, Journal of crystal growth, 2005, 284, 517-529.

25-N. K. Shapur, V. Uvarov, I. Popov, R. Katz, O. N. Gofrit, E. H. Landau, D. Pode, M. Duvdevani, Crystallite size - Is it a new predictor for renal stone burden?, Urology, 2012, 80, 980-985.

26- A. Alaya, A. Nouri, M. Belgith, H. Saad, I. Hell, W. Hellara, R. Jouini, M. F. Najjar, Changes in kidney stones type according to sex and age in Tunisian patients, Actas Urológicas Españolas (English Edition), 2012, 36, 171-177.
27-M. Daudon, O. Traxer, E. Lechevallier, C. Saussine, Epidemiology of urolithiasis, Progres en urologie: journal de l'Association francaise d'urologie et de la Societe francaise d'urologie, 2008, 18, 802-814.

28-M. Daudon, Comment analyser un calcul et comment interpréter le résultat, L'Eurobiologiste (Paris), 1993, 27, 35-46.

29-B. El Guerrouj, M. Bouhrim, Y. Bentata, M. Daudon, M. Melhaoui, L. Kharchoufa, N. Bencheikh, O. Bekkouch, Kidney Stone Disease (Urolithiasis): Epidemiological Study in the Eastern Region of Morocco, European Journal of Scientific Research, 2019, 155, 40-57.

30-D. B. Leusmann, R. Blaschke, W. Schmandt, Results of 5035 stone analyses: a contribution to epidemiology of urinary stone disease, Scandinavian journal of urology and nephrology, 1990, 24, 205-210.

31-T. Koide, T. Oka, M. Takaha, T. Sonoda, Urinary tract stone disease in modern Japan, European urology, 1986, 12, 403-407. 